\title{
Identificación, Análisis y Evaluación del Riesgo de Exposición Ocupacional en Servicios de Preparación de Citostáticos de la Red Nacional de Prestadores
}

\author{
IDENTIFICATION, ANALYSIS AND RISK ASSESSMENT OF OCCUPATIONAL EXPOSURE IN CYTOSTATIC PREPARATION \\ SERVICES OF THE PROVIDERS NATIONAL NETWORK
}

\author{
Esteban Villarroel Cantillana \\ Ingeniero Civil Bioquímico, Magíster en Salud Pública. Asociación Chilena de Seguridad, Santiago de Chile.
}

\section{RESUMEN}

Se identifica, analiza y evalúa el riesgo de exposición ocupacional a citostáticos, en una muestra de instalaciones destinadas a preparación de mezclas destinadas a tratamiento terapéutico oncológico, pertenecientes a la Red Nacional de Prestadores autorizados; en relación con el tipo y nivel de infraestructura de control y frecuencia de exposición asociada. Los resultados de la evaluación revelan una gran variedad de situaciones en las que, pese a la existencia de una regulación que define el tipo y caracteristicas de la contención mínima necesaria, se observan insuficiencias en el nivel de control observado y consiguiente riesgo al que se expone el personal.

Del total de instalaciones incluidas en el estudio, sólo el 7\% presenta un nivel de control aceptable y cercano al óptimo esperado, el 43\% presenta un nivel de control regular y mejorable a través de correcciones menores. En tanto el 50\% restante, revela un significativo nivel de insuficiencia; destacando que el 36\% de las instalaciones evidencia condiciones extremas de exposición laboral.

(Villarroel E, 2014. Identificación, Análisis y Evaluación del Riesgo de Exposición Ocupacional en Servicios de Preparación de Citostáticos de la Red Nacional de Prestadores. Cienc Trab. Ene-Abr; 16 [49]: 56-64).

Palabras Clave: EXPOSICIÓN OCUPACIONAL, CITOSTÁTICO, CITOTÓXICO.

\section{ABSTRACT}

The risk of occupational exposure to cytostatic was identified, analyzed and evaluated, in a sample of facilities used for preparation of mixtures for oncological therapeutic treatment, belonging to the National Network of Authorized Providers; regarding the type and level of infrastructure of control and frequency of associated exposure. The results of the evaluation reveal a great variety of situations where, despite the existence of a regulation that defines the type and characteristics of the minimum containment required, gaps are observed in the observed level of control and consequent risk which personnel is exposed to.

From the total number of facilities included in the study, only $7 \%$ have a level of control acceptable and close to the optimal that is expected, 43\% present a control level that is regular and improvable through minor corrections. Meanwhile, the remaining 50\% show a significant level of insufficiency; it is underlined that 36\% of facilities evidence extreme occupational exposure conditions.

Key words: OCCUPATIONAL EXPOSURE, CYTOSTATIC, CYTOTOXIC.

\section{INTRODUCCIÓN}

La manipulación, preparación y administración de drogas citostáticas (citotóxicos), por procedimientos asociados a manejo de soluciones (apertura de ampollas, vertido, agitación mecánica, trasvasije, dosificación y descarte), genera una serie de riesgos asociados a la inhalación de aerosoles y posibles contactos directos con la piel debido a derrames y/o salpicaduras. En este sentido, desde que fue reconocido el hecho que la exposición ocupacional a los agentes citados, representa un riesgo para la salud de los trabajadores que los manipulan, una serie de estudios

Correspondencia / Correspondence:

Esteban Villarroel Cantillana

Ramón Carnicer 163, Providencia. Santiago, Chile.

Tel.: (02) 25157556

e-mail: evillarroel@achs.cl

Recibido: 17 de Febrero de 2014 / Aceptado: 18 de Marzo de 2014 han examinado los efectos fisiológicos relacionados con la exposición profesional ${ }^{1-7}$; sin embargo, ninguno de ellos evidencia la existencia de un indicador biológico, con las condiciones necesarias para ser utilizado como herramienta de vigilancia epidemiológica que determine una correlación evidente entre exposición y efecto fisiológico, producto de una exposición laboral a este tipo de agentes.

Desde el punto de vista preventivo, al no mediar las condiciones mínimas necesarias que eviten el contacto o minimicen la exposición, el trabajador expuesto a tales sustancias, se encuentra bajo una condición de riesgo laboral, cuya extensión en el tiempo, puede generar condiciones que favorezcan el desarrollo de tejido neoplásico, haciendo de ésta una posible afección desarrollada de una manera directa por el ejercicio de la profesión o el trabajo, con resultado de incapacidad o muerte. A ello se suma el hecho que tampoco se cuenta con normativa referente a límites de exposición laboral ambiental, condición asociada a la complejidad de control sobre variables relativas a la sensibilidad y especificidad en relación con grados de absorción biológica, sub registros históricos de exposición y variabilidad de la exposición, en relación a los medios de control, variedad y características de las sustancias utilizadas; 
variables que en su conjunto limitan la identificación de un indicador biológico de exposición. Sin pasar por alto, que dicho criterio apunta a determinar una condición de post contacto, que evalúa el efecto una vez que la exposición ya ha ocurrido; punto de vista que se contrapone a un criterio de carácter preventivo.

En razón de lo anterior, resulta razonable la necesidad de determinar, mediante la realizacion de un estudio de campo, si las condiciones de control, en una muestra de instalaciones autorizadas, destinadas a la preparación de citostáticos, corresponden a las mínimas necesarias que demanda el trabajo seguro con este tipo agentes, de acuerdo a los criterios y lineamientos generales establecidos en la normativa referencial reconocida y exigida por el Ministerio de Salud y otros organismos referenciales. ${ }^{8-12}$ De acuerdo a ello, se establece como hipótesis de trabajo, que las instalaciones autorizadas, destinadas a la preparación de citostáticos, pertenecientes a los Servicios de Preparación de Citostáticos de la Red Nacional de Prestadores (públicos y/o privados), no poseen el nivel de control mínimo necesario para controlar la exposición laboral asociada a la manipulación segura de este tipo de agentes, correspondiendo el objetivo principal de la investigación, además de la evaluar la condición de exposición laboral existente en el país, diseñar y aplicar una metodología de evaluación del nivel de exposición laboral, metodología basada en la aplicación de criterios preventivos que permitan asegurar un margen significativo de control sobre el riesgo de exposición.

\section{METODOLOGÍA DE EVALUACIÓN DEL RIESGO DE EXPOSICION LABORAL}

En el marco del desarrollo de la investigación, se invitó a participar de ésta al conjunto de 43 instalaciones, pertenecientes a la Red Nacional de Prestadores autorizados para realizar dicha actividad por el Ministerio de Salud, ello a través del envío de carta formal dirigida a cada uno de los Directores y/o Representante legal correspondiente, invitación aceptada por 14 de las 43 instituciones autorizadas, representantes del 32\% del total, correspondientes a clínicas privadas, hospitales públicos y universitarios, institutos y fundaciones, distribuidas en diferentes áreas geográficas del país: 7 en la Región Metropolitana, 4 ubicadas en regiones próximas a ésta (IV, V, VI) y 3 ubicadas en regiones alejadas (VIII, XII y XIV); cuya cobertura de prestación, asociada a tratamiento quimioterapéutico por diagnóstico de cáncer, satisface las necesidades del 70\% de la población nacional.

Confirmada la intención de participar del estudio, se procedió a programar y realizar reuniones de trabajo y vista a las instalaciones destinadas a preparación de citostáticos, a fin de aplicar la herramienta de evaluación diseñada; información con la que se procedió a la elaboración de Informe de evaluación para cada institución, cual da cuenta del estado situacional de la instalación al momento de la visita, en relación con la normativa nacional vigente e internacional atingente, considerando antecedentes respecto de la actividad, agentes de riesgo, su efecto en las personas, descripción de los sistemas de control observados, metodología de evaluación utilizada, resultados de la evaluación aplicada, análisis correspondiente, conclusiones y recomendaciones atingentes, en relación a los hallazgos observados.

La metodología utilizada, consideró la estructuración de un procedimiento de evaluación de las condiciones de funcionamiento de las instalaciones, desde un punto de vista integral; lo que incluyó evaluar la infraestructura y contención existente, en relación con la selección de la tecnología, su uso y estado de funcionamiento y la forma en la cual se logra su efecto interactivo (tipo y frecuencia de exposición).

\section{Riesgo de Exposición Ocupacional. Identificación y Análisis del Nivel de Contención}

La evaluación de la exposición y riesgo asociado, se realizó mediante la aplicación de un algoritmo, estructurado sobre la base del desarrollo de una metodología basada en la aplicación de matrices de verificación que consideran una descripción de las condiciones mínimas de contención que aseguren el control sobre las diferentes tareas que incluyen exposición a los riesgos propios de la manipulación de sustancias citotóxicas. En este sentido, se definió como condición esperada aquella que da cuenta del cumplimiento de todas las características de contención necesarias para el control efectivo de la exposición y sus riesgos; cuya comparación con la realidad observada (condición verificada) permitió establecer un valor relativo de control, en relación con el nivel de logro alcanzado.

De acuerdo a lo señalado, existirian una serie de condiciones mínimas de contención a considerar dentro del estudio de una actividad o rubro, cuyo análisis requiere agruparlas, en orden a facilitar el procesamiento de las mismas en matrices de verificación, y de esta forma, estableciendo factores de ponderación para cada una y el puntaje correspondiente, en función de la importancia que se les asigna dentro del nivel de contención particular y global que debe considerar el control efectivo de la exposición y sus riesgos; ponderación que permitirá obtener su valor real, $R$, basado en la existencia de los medios de contención necesarios y representativos del nivel de control o contención global en la instalación.

Para obtener lo anterior fue necesario establecer en base a los requisitos normados, recomendados y aplicables al trabajo en estudio, los factores relevantes para su ejecución de manera controlada, que constituirán cada matriz en particular; a cada una de las cuales se les asignó una ponderación de acuerdo a su importancia relativa $\left(X_{i}\right)$ en el contexto global, cuya suma como máximo alcanza el valor 1 . Asimismo, cada matriz consideró la desagregación del factor que representa en sus ítem diferenciables más importantes, que permitieron objetivar la evaluación del factor, asignándole puntajes ponderados por su importancia relativa en su aporte al control o contención del factor, situación en la cual el detalle, reconocimiento y verificación de la condición esperada permitió establecer un puntaje por matriz, $M_{i}$.

Para dicho efecto se consideró la utilización de 7 matrices elaboradas y destinadas a verificar la existencia y uso de condiciones asociadas a aspectos de ubicación y acceso, características constructivas (sellos y acabados), elementos de control ambiental, medios de contención perimetral, condiciones necesarias y relativas a los servicios de asistencia y apoyo, integridad estructural del nivel de contención y aquel relativo a capacitación y adquisición de competencias de los trabajadores. En este sentido, tal como fuera señalado, el cumplimiento de los requisitos, representa el cumplimiento del nivel de contención y control mínimo necesario que demanda el nivel de frecuencia con el cual se expone el trabajador a la actividad

De esta forma, el puntaje obtenido en cada matriz, expresado en forma porcentual, equivale al nivel de cumplimiento de los items 
Tabla 1.

Escala según Valor Relativo del Nivel de Contención.

\begin{tabular}{|lc|} 
Rango $R$ & Escala \\
$0,00-0,25$ & 4 \\
$0,25-0,50$ & 3 \\
$0,50-0,75$ & 2 \\
$0,75-1,00$ & 1 \\
\hline
\end{tabular}

de cada factor en su correspondiente matriz, que aplicado a la ponderación del factor en la contención global $\left(X_{i}\right)$, permitió obtener el nivel de control aportado. La sumatoria de los valores obtenidos para cada factor y su ponderación respectiva permitió obtener el nivel de contención real global existente, $R$.

Como es de esperar, el valor de $R$ obtenido presenta valores que varían en el rango entre 0 y 1 , lo que permite diferenciar tramos en relación con su nivel en relación con una escala como la que se presenta en la tabla $\mathrm{N}^{\circ} 1$, en la cual el mayor valor de la escala corresponde al rango de valor $\mathrm{R}$ obtenido que representa una condición de control o contención más baja y viceversa para el valor menor. En este caso, se considera que una instalación posee un nivel de contención aceptable, cuando su $R_{i}$ sea mayor o igual a 0,75 (valor escala 1 )

En el caso en estudio, las matrices de verificación señaladas, consideraron aspectos cualitativos relativos a ubicación y acceso a la instalación, características constructivas, elementos de control ambiental, servicios de apoyo y asistencia, integridad estructural y medios de contención existentes, así como las acciones dirigidas a capacitación y adquisición de competencias realizadas por los trabajadores involucrados. De esta forma el reconocimiento de cada elemento verificador, suma puntaje la matriz respectiva, en relación a una ponderación interna y esta respecto del total, valoración que finalmente asigna un valor numeral característico posible de incluir en alguno de los intervalos definidos en la tabla $\mathrm{N}^{\circ} 1$.

\section{Riesgo de Exposición Ocupacional asociado a Frecuencia de Exposición.}

Establecido el valor correspondiente dentro de la escala de la tabla $\mathrm{N}^{\circ} 1$, asociado al nivel de contención existente en la instalación, es necesario establecer el nivel de exposición laboral asociado al contacto con este tipo de agentes, lo que es posible de determinar, mediante el cálculo del denominado Índice de Contacto Citotóxico (ICC), valor que permite estimar la intensidad del contacto como valor indicativo y con un criterio objetivo de los niveles de exposición, en relación con la actividad del trabajador, asociada a la extensión de la jornada, número de preparaciones y administraciones realizadas en un período definido, de acuerdo a la siguiente expresión, adaptada de la reerencia original ( ).

$$
I C C=\begin{gathered}
N_{P}+N_{A}+N_{R} \\
N_{H}
\end{gathered}
$$

Donde:

ICC: Índice de contacto citotóxico.

$\mathrm{N}_{\mathrm{P}}$ : Número de preparaciones realizadas por una misma persona durante la semana laboral.

$\mathrm{N}_{\mathrm{A}}$ : Número de administraciones realizadas por una misma persona durante la semana laboral.

$\mathrm{N}_{\mathrm{R}}$ : Número de retiros realizadas por una misma persona durante la semana laboral.

$\mathrm{N}_{\mathrm{H}}$ : Número de horas invertidas en la tarea por la persona durante la semana laboral.
El indice define tres niveles crecientes de exposición:

- Nivel 1, ICC < 1: Corresponde a la preparación y administración ocasional.

- Nivel 2, $1<$ ICC < 3: Corresponde a la preparación y administración de cantidades moderadas.

- Nivel 3, ICC > 3: Corresponde a la preparación y administración de manera intensiva y rutinaria.

Calificación del Nivel de Riesgo de Exposición Ocupacional

Calculado el Índice de contacto citotóxico, se procede a multiplicar el nivel de exposición asociado al índice de contacto citotóxico por el valor escala, correspondiente al rango en el cual se encuentra el valor relativo asociado al nivel de contención existente en la instalación. Esta operación permite obtener un valor referencial en el rango entre 1 y 12, cuya disposición en una matriz como la que se presenta en la tabla $\mathrm{N}^{\circ} 2$, permite correlacionar el nivel de contención existente, indice de contacto citotóxico y nivel de exposición en las condiciones de trabajo observadas. Tabla en la que, además, es posible establecer una escala conceptual, basada en un código de colores, permitiendo, junto a la identificación de una condición de riesgo, establecer la necesidad de desarrollar acciones a fin de superar las brechas observadas.

Tabla 2.

Criterio de Calificación del Nivel de Riesgo Trabajadores Expuestos a Agentes Citotóxicos.

\begin{tabular}{|c|c|c|c|}
\hline Escala, según R & \multicolumn{4}{|c|}{ Índice Contacto Citotóxico } \\
\hline $\mathbf{4}$ & $\mathbf{1}$ & $\mathbf{2}$ & $\mathbf{3}$ \\
\hline $\mathbf{3}$ & $\mathbf{4}$ & 8 & 12 \\
$\mathbf{2}$ & $\mathbf{3}$ & 6 & 9 \\
$\mathbf{1}$ & 2 & 4 & 6 \\
\hline
\end{tabular}

Nivel de Riesgo 1, riesgo bajo.

Nivel de Riesgo 2, riesgo medio.

Nivel de Riesgo 3, riesgo alto.

Donde:

- El Nivel 1 corresponde al trabajo desarrollado en condiciones de riesgo bajo, condición laboral representativa de una exposición controlada y no representativa de riesgo de una enfermedad atribuible al ejercicio profesional, derivada de la exposición a agentes citostáticos (citotóxicos).

- El Nivel 2 corresponde al trabajo desarrollado en condiciones de riesgo medio, condición laboral representativa de una actividad cuya realización incluye la presencia del riesgo debido a la falta de una gestión que incluya acciones de control sobre el riesgo y la exposición, condición que pudiera favorecer la existencia de casos de enfermos profesionales. Se requiere de evaluación médica competente para la detección y diagnóstico de posibles efectos en la salud de las personas y el análisis de las falencias existentes, respecto de las matrices de verificación correspondientes.

- El Nivel 3 corresponde al trabajo desarrollado en condiciones de riesgo alto, condición laboral en la cual la gestión sobre el riesgo y la exposición es mínima, representando un claro riesgo para la salud de los trabajadores. Se requiere de la inmediata evaluación médica competente para la detección y diagnóstico de posibles efectos en la salud de las personas, junto al análisis e implementación de las falencias existentes, respecto de las matrices de verificación correspondientes. 
Tabla 3.

Resultados Valoración entre la realidad observada y esperada, Mi/MEi. Instalaciones destinadas a Área de Preparaciones de la Red Nacional de Prestadores.

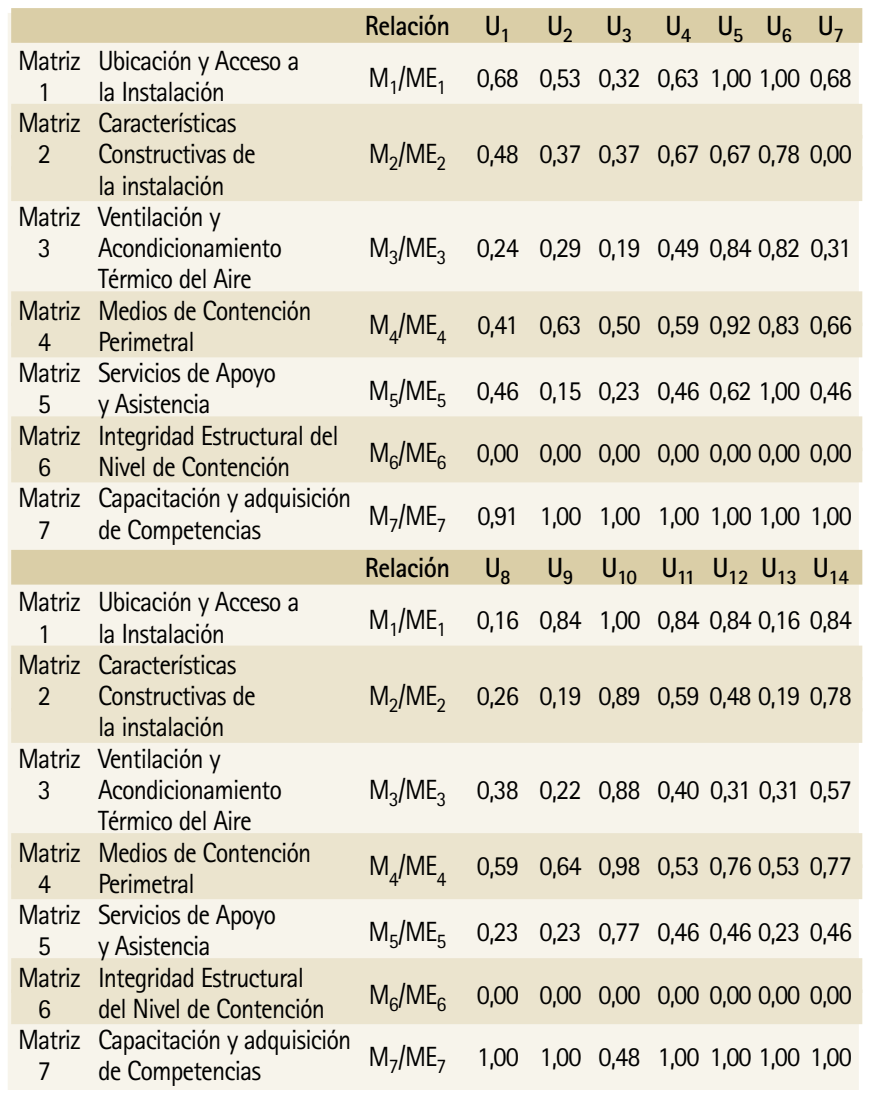

Figura 2.

Cumplimiento del Nivel de Contención Global en Instalaciones destinadas a Preparación de Citotóxicos en la Red Nacional de Prestadores.

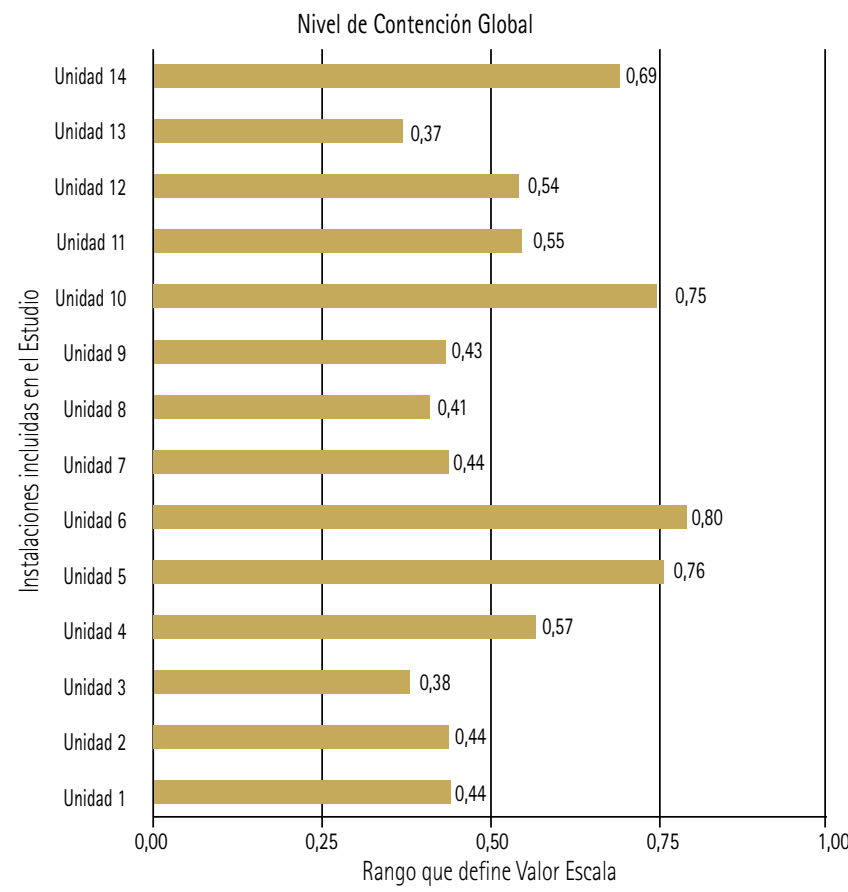

Figura 3.

Determinación Índice de Contacto Citotóxico, ICC, en Instalaciones destinadas a Área de Preparaciones de la Red Nacional de Prestadores.

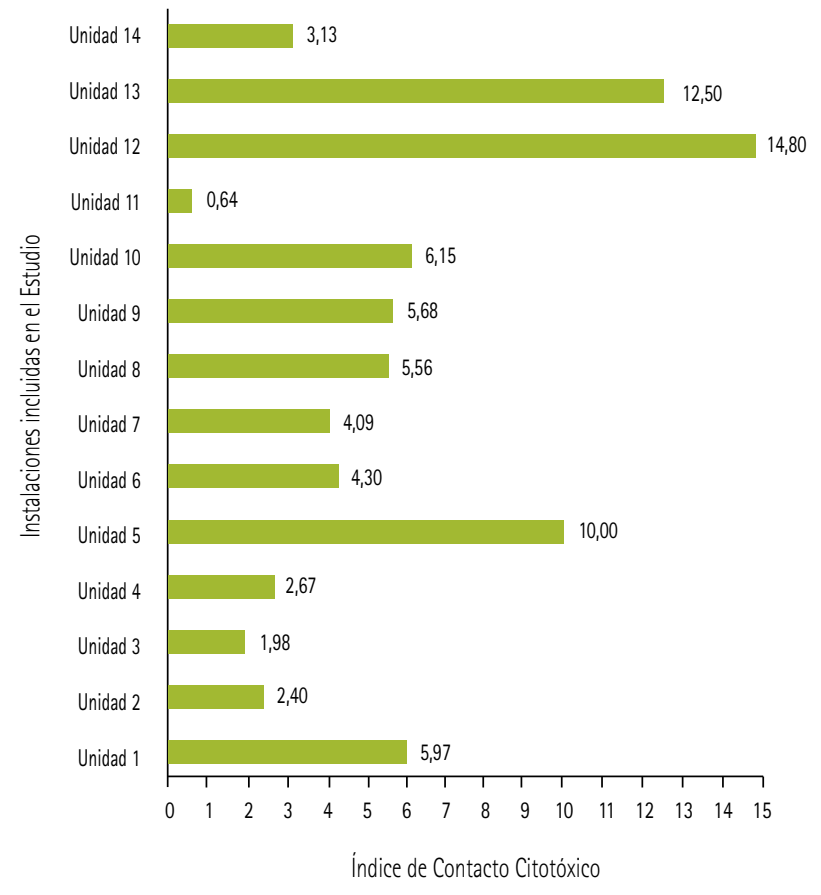

\section{RESULTADOS}

\section{Riesgo de Exposición Ocupacional. Identificación y Análisis del Nivel de Contención}

De acuerdo al algoritmo descrito y en base a la aplicación de la herramienta de evaluación señalada, fue posible establecer en cada Unidad, la relación existente entre la condición observada en terreno y aquella óptima esperada, $\mathrm{Mi}_{\mathrm{i}} / \mathrm{ME}_{\mathrm{i}}$, resultados que se presentan en la tabla No3, donde $\mathrm{U}_{\mathrm{i}}$, identifica a la Unidad perteneciente a la instalación, destinada a preparación de citostáticos u otras sustancias con efecto citotóxico. Como se desprende de la tabla №3, ninguna de las instalaciones evidenció de aplicación de algún tipo de test que asegure y/o verifique la integridad estructural del nivel de contención existente, que para fines del estudio se detalla en la Matriz №6.

\section{Riesgo de Exposición Ocupacional. Nivel de Contención}

Determinada la relación entre la condición óptima esperada y aquella observada en terreno, mediante aplicación de ponderaciones internas y relación entre las diferentes matrices, se procedió a determinar en cada caso el nivel de contención global individual, $R_{i}$, correspondiente a cada instalación, cuyo resumen se presenta en la figura $\mathrm{N}^{\circ} 2$.

\section{Riesgo de Exposición Ocupacional asociado a Frecuencia de Exposición. Nivel de Exposición}

De acuerdo a la información señalada por cada una las instituciones, en relación al número de preparaciones promedio mensual, número de trabajadores involucrados en tareas de preparación, extensión de la jornada individual y horas asignadas a tareas de preparación, se procedió a determinar el índice de contacto citotóxico, como indicador relativo de la frecuencia de exposición a la cual expone el trabajo realizado, cuyo detalle se presenta en la figura №3. 
Tabla 6.

Valor Escala asociada al resultado de la Evaluación del Nivel de Contención observado.

\begin{tabular}{|ccc} 
& Contención Global, $\mathbf{R}_{\mathrm{i}}$ & Valor Escala \\
\hline Unidad 1 & 0,44 & 3 \\
Unidad 2 & 0,44 & 3 \\
Unidad 3 & 0,38 & 3 \\
Unidad 4 & 0,57 & 2 \\
Unidad 5 & 0,76 & 1 \\
Unidad 6 & 0,80 & 1 \\
Unidad 7 & 0,44 & 3 \\
Unidad 8 & 0,41 & 3 \\
Unidad 9 & 0,43 & 3 \\
Unidad 10 & 0,75 & 1 \\
Unidad 11 & 0,55 & 2 \\
Unidad 12 & 0,54 & 2 \\
Unidad 13 & 0,38 & 3 \\
Unidad 14 & 0,69 & 2
\end{tabular}

Figura 4.

Distribución Porcentual Instalaciones.

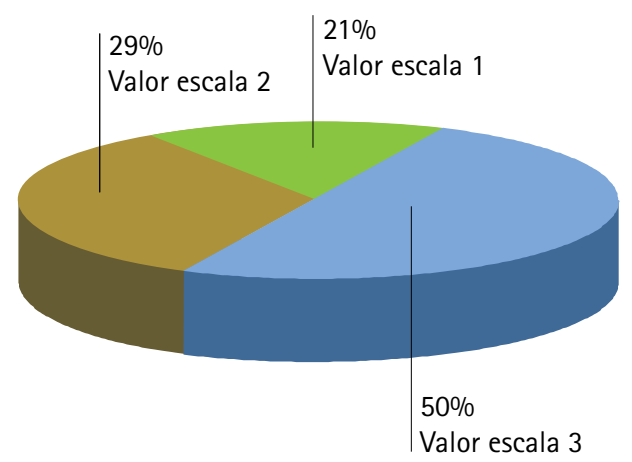

\section{ANÁLISIS Y CONCLUSIONES}

\section{Riesgo de Exposición Ocupacional. Identificación y Análisis del Nivel de Contención Global}

Realizado el algoritmo de cálculo correspondiente, resultado de las ponderaciones internas y entre las diferentes matrices, cuyos valores se resumen en la tabla $\mathrm{N}^{\circ} 4$ y figura $\mathrm{N}^{\circ} 4$, se observa que sólo 3 de las instalaciones, Unidad 5, Unidad 6 y Unidad 10, representativas del $21 \%$ del total, cumplen con un nivel de contención y capacitación suficiente que garantiza el control de la exposición derivada de las tareas que involucran preparación de drogas terapéuticas oncológicas (valor escala 1). En tanto, 4 de las instalaciones (29\% del total), presentan un nivel de contención medio superior (valor escala 2). El resto de las instalaciones, 7, representativas del 50\% del total, presentan un nivel de contención medio inferior (valor escala 3). No se observan instalaciones con nivel de contención extremo inferior (valor escala 4).

De lo que se infiere que, el 78,6\% de las instalaciones incluidas en el estudio, no poseen, en relación a los medios existentes, un nivel de infraestructura suficiente para controlar la exposición laboral asociada a la preparación de citostáticos.

\section{Riesgo Exposición Ocupacional asociado a Frecuencia de Exposición}

De acuerdo a información señalada por cada institución, el índice de contacto citotóxico para los trabajadores que realizan tareas asociadas a preparación de citostáticos, presenta una amplia varia-
Tabla 8.

Nivel de Riesgo asociado a Frecuencia de Exposición, ICC.

$\begin{array}{lcc} & \text { ICC }_{\mathbf{i}} & \text { Nivel de Riesgo } \\ \text { Unidad 1 } & 5,97 & 3 \\ \text { Unidad 2 } & 2,40 & 2 \\ \text { Unidad 3 } & 1,98 & 2 \\ \text { Unidad 4 } & 2,67 & 2 \\ \text { Unidad 5 } & 10,00 & 3 \\ \text { Unidad 6 } & 4,30 & 3 \\ \text { Unidad 7 } & 4,09 & 3 \\ \text { Unidad 8 } & 5,56 & 3 \\ \text { Unidad 9 } & 5,68 & 3 \\ \text { Unidad 10 } & 6,15 & 3 \\ \text { Unidad 11 } & 0,64 & 1 \\ \text { Unidad 12 } & 14,80 & 3 \\ \text { Unidad 13 } & 12,50 & 3 \\ \text { Unidad 14 } & 3,13 & 3\end{array}$

Figura 6.

Distribución Porcentual ICC en Instalaciones.

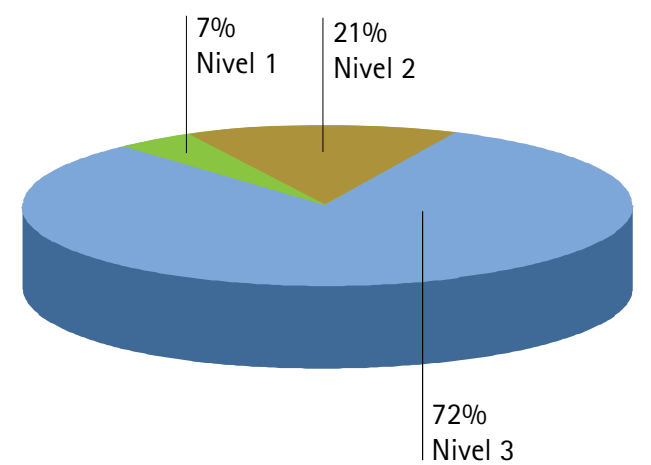

ción, la que incluye todos los niveles de riesgo considerados en la definición del mismo. En la tabla $\mathrm{N}^{\circ} 5$ y figura $\mathrm{N}^{\circ} 5$ se observa que en sólo una de las instalaciones, Unidad 11, correspondiente al 7\% del total de la muestra, la combinación entre el número de profesionales involucrados de manera directa y horas asignadas a tareas de preparación, representa un nivel de riesgo bajo, asociado a la frecuencia de exposición.

En tanto, en el resto de las instalaciones, 93\% del total de la muestra, se observa que dicha combinación no es suficiente para controlar el riesgo asociado a frecuencia de exposición, debiendo destacar que de éste valor, el 72\% presenta una condición extrema (Nivel 3) y el 21\% restante, una condición riesgosa intermedia por este concepto (Nivel 2).

\section{Calificación del Nivel de Riesgo de Exposición Ocupacional} Calculado el Índice de Contacto Citotóxico, se procede a multiplicar el nivel de exposición asociado al índice de contacto citotóxico por el valor escala correspondiente al rango en el cual se encuentra el valor relativo asociado al nivel de contención existente en la instalación, y de esta forma establecer el Nivel de Riesgo de Exposición Ocupacional al que se exponen los trabajadores, en relación a la contención existente y frecuencia de exposición. El detalle global se presenta en la tabla №9 y figura $\mathrm{N}^{\circ} 7$, en las que se observa que en el 93\% de las instalaciones existen condiciones de riesgo no controladas, en relación a la combinación entre contención y frecuencia de exposición (50\% con riesgo alto y 43\% con riesgo medio), y que sólo en el 7\% de ellas se observa condiciones de riesgo bajo, en relación 
Tabla 9.

Nivel de Riesgo de Exposición Ocupacional. Resumen Resultados.

$\begin{array}{lccc}\text { Valor Escala, } & \begin{array}{c}\text { Nivel de Exposición, } \\ \text { según ICCi }\end{array} & \begin{array}{c}\text { Nivel } \\ \text { de Riesgo }\end{array} \\ \text { Unidad 1 } & 3 & 3 & 9 \\ \text { Unidad 2 } & 3 & 2 & 6 \\ \text { Unidad 3 } & 3 & 2 & 6 \\ \text { Unidad 4 } & 2 & 2 & 4 \\ \text { Unidad 5 } & 1 & 3 & 3 \\ \text { Unidad 6 } & 1 & 3 & 3 \\ \text { Unidad 7 } & 3 & 3 & 9 \\ \text { Unidad 8 } & 3 & 3 & 9 \\ \text { Unidad 9 } & 3 & 3 & 9 \\ \text { Unidad 10 } & 1 & 3 & 4 \\ \text { Unidad 11 } & 2 & 1 & 2 \\ \text { Unidad 12 } & 2 & 3 & 6 \\ \text { Unidad 13 } & 3 & 3 & 9 \\ \text { Unidad 14 } & 2 & 3 & 6\end{array}$

Figura 7.

Nivel de Riesgo de Exposición Ocupacional.

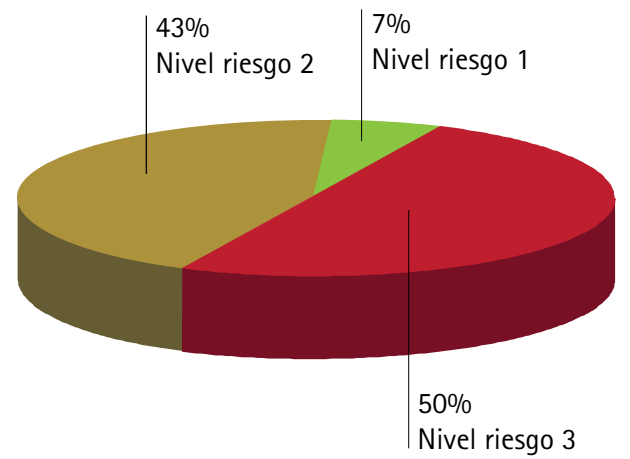

a la existencia y suficiencia de los medios, frecuencia e idoneidad del personal. Lo anterior implica que solo el 7\% presenta una condición de exposición laboral controlada y no representativa de riesgo de una enfermedad profesional derivada de la exposición a agentes citostáticos (citotóxicos).

\section{CONCLUSIONES GENERALES DEL ESTUDIO}

En términos generales, considerando una proyección de los resultados, se concluye que:

\section{Determinación del Nivel de Contención Global}

En relación a la contención global existente, sólo el 21\% del total, cumple con un nivel de contención y capacitación suficiente y necesaria que garantice, por este medio, el control de la exposición derivada de las tareas que involucran preparación de drogas terapéuticas oncológicas (Valor $\mathrm{R} \mathrm{i}$, igual o superior a 0,75 ).

\section{Determinación del Nivel de Exposición asociado a Frecuencia de Exposición}

En relación a la frecuencia de exposición, en sólo el 7\% del total de la muestra, la combinación entre el número de profesionales involucrados de manera directa y horas asignadas a tareas de preparación, representa un nivel de riesgo bajo (se considera un nivel de riesgo bajo, valores ICC $\mathrm{i}$, menores a $1 \mathrm{y}$ nivel de riesgo alto, valores de ICC $\mathrm{i}$, mayores a 3).

En tanto, en el resto de las instalaciones, 93\% del total, se observa que dicha combinación no es suficiente para controlar el riesgo asociado a frecuencia de exposición; destacando que en el 72\% de la muestra, se observa una condición de exposición extrema por éste factor (Valor ICCi, igual o superior a 3).

\section{Determinación del Nivel de Riesgo de Exposición Ocupacional a Citostáticos}

En relación a la combinación entre contención y frecuencia de exposición, se observa que en el 93\% de las instalaciones existen condiciones de riesgo no controladas. En un 43\% de ellas, se observa un nivel de control regular, mejorable a través de correcciones menores, en relación a la regularización de sistemas de control ya existentes, y en el 50\% restante, se observa un nivel de insuficiencia importante. Destacando que el 36\% de las instalaciones evidencia condiciones extremas de exposición, asociadas a la insuficiencia de medios y alejamiento del nivel óptimo, situación que en algunos casos, ha motivado la contratación del servicio de preparación a terceros y/o cierre temporal.

Sólo en el 7\% de las instalaciones, se observan condiciones de riesgo bajo, en relación a la existencia y suficiencia de los medios, frecuencia e idoneidad del personal (Unidad 11, Nivel de Riesgo 1).

\section{RECOMENDACIONES}

\section{Nivel de Riesgo asociado al Control a Nivel de Infraestructura y Capacitación}

La condición observada revela que las principales brechas asociadas a contención, se relacionan con insuficiencias en los medios asociados a diseño, instalación u operación de los servicios de apoyo y asistencia (matriz $\mathrm{N}^{\circ} 5$ ), uso e implementación de sistemas de control aerodinámico, general y localizados (matriz $\mathrm{N}^{\circ} 3$ ) y aquellos referidos a características constructivas de la instalación terminaciones, tipo de materiales (matriz $\mathrm{N}^{\circ} 2$ ) y acabados según solicitaciones. Por ello, las recomendaciones son aplicables a un porcentaje importante de las instalaciones, en especial aquellas que se encuentran en un rango de control medio superior, definido como aquel representativo de niveles de logro entre 50 y 75\%. En cuyo caso, las instituciones involucradas deberán considerar mejoras en aquellos items de las matrices señaladas, cuya evaluación evidenció no existencia o no apego a la normativa referencial.

Destaca la necesidad de priorizar la regularización del funcionamiento de las respectivas cabinas de seguridad biológicas instaladas, según origen, licencia y normativa aplicable, segregación aerodinámica respecto de áreas relacionadas, incluyendo implementación y verificación de los medios que aseguren estanqueidad, calidad y cantidad de aire necesario para asegurar el control de la contaminación y exposición (matrices $\mathrm{N}^{\circ} 6 \mathrm{y} \mathrm{N}^{\circ}$ ), así como los medios de respaldo de energía que su funcionamiento requiere, desde el punto de vista de la criticidad de la operación y seguridad de las personas. En este sentido, uno de los hallazgos importantes de considerar, es el hecho que aun cuando existe un conocimiento de la normativa aplicable, las bases técnicas de licitación que contratan el servicio de verificación del estado de funcionamiento a terceros, no incluyen de manera destacada la necesidad de aplicar los diferentes test que corresponde al tipo y modelo de cabina de seguridad biológica instalada, observando una clara falta de conocimiento del detalle necesario por parte del área administrativa de la Institución, respecto de las necesidades del área de operaciones. A ello se suma el hecho que un análisis del registro histórico de dichas 
bases, revela que por un problema presupuestario, la complejidad de las mismas ha disminuido con el tiempo (mayor número de test, incrementa el valor del servicio) y no se observa la necesaria acción de contratación de una contraparte técnica para verificar alguna de las evaluaciones realizadas. En este contexto, en al menos tres oportunidades, se observó un claro desfase entre la fecha de medición y la fecha de entrega del informe correspondiente, situación atribuible a la falta de recursos para realizar el pago de la prestación, plazo que en uno de los casos superó los 12 meses, es decir, un total desconocimiento del estado situacional de la instalación por un período de 24 meses.

Debiendo destacar que las principales falencias observadas, coinciden con los items de mayor criticidad, en el sentido del logro efectivo de la necesaria contención requerida, en particular aquellos representados por las matrices $\mathrm{N}^{\circ} 3$ y $\mathrm{N}^{\circ} 2$, cuya valoración relativa suma el 45\% del total. Por lo anterior, la asignación de los recursos necesarios para su resolución, tendrá directa implicancia en la mejora del nivel de contención observado.

En lo que respecta al ítem asociado a capacitación y adquisición de competencias (matriz $\mathrm{N}^{\circ}$ ), aun cuando se observa un nivel importante de logro, se recomienda formalizar la sistematización de un programa de capacitación y contenidos, evaluable y de aplicación periódica, que considere aspectos tecnológicos, procedimentales, seguridad y riesgos ocupacionales asociados. Esto es especialmente necesario al momento de existir modificaciones en la regulación, interpretación y aplicación de la normativa existente y aquella en revisión.

\section{Nivel de Riesgo asociado a Frecuencia de Exposición}

La condición observada revela la existencia de una amplia variedad de combinaciones entre el número de profesionales involucrados directamente en tareas de preparación y horas asignadas a dicha actividad, generando valores de índice de contacto citotóxico en los diferentes rangos definidos, incluyendo valores significativamente altos, ya que en solo el $28 \%$ de las instalaciones se observan ICC $i$ menor a 3. Por ello se recomienda realizar las gestiones administrativas necesarias, para disminuir este factor de riesgo, ya sea por la vía de una distribución de carga de trabajo homogénea en el actual número de trabajadores y/o aumento en el número de trabajadores con las competencias necesarias para dicha actividad. Representando una alternativa recomendable, establecer que ningún trabajador deberá estar expuesto a actividades que generen valores de ICC $_{\mathrm{i}}$ mayores a 4,5; factor complementario a la condicion de control asociada a que la evaluación de contención y capacitación existente logre valores en el rango superior, $\mathrm{R}_{\mathrm{i}}>0,75$.

\section{Nivel de Riesgo asociado a la Exposición Ocupacional a Citostáticos}

El análisis de situación, evidencia la existencia de importantes brechas a nivel de contención y frecuencia de exposición observada, las que en su conjunto revelan que en el 93\% de las instalaciones existen condiciones de riesgo no controladas, que en la mayoría de los casos se atribuye a la no existencia y/o aplicación de las acciones administrativas necesarias para disminuir el riesgo asociado a frecuencia de exposición, relativas a disminuir el número de preparaciones realizadas, reducir carga de trabajo productiva y/o aumentar el número de trabajadores involucrados ( ). En menor grado, pero no menos importante, un factor coadyuvante es la ausencia de medios ingenieriles o la necesaria y efectiva gestión sobre el funcionamiento de los mismos, en relación a asegurar la suficiencia de éstos para controlar las características que demanda la calidad del proceso y exposición ocupacional derivada de las tareas realizadas, en particular la parcialidad o no existencia de un programa de mantención preventiva, capaz de verificar de manera organizada y sistemática el cumplimiento de los parámetros operacionales de los diferentes equipos y sistemas de control instalados.

En razón de lo anterior, se recomienda estructurar un protocolo de evaluación y/o verificación del estado de funcionamiento de este tipo de instalaciones, el que deberá ser realizado por terceros con experiencia y conocimiento necesarios, que considere aspectos normativos, metodológicos e instrumentales aplicables a las diferentes tecnologías implementadas para el control de las diferentes variables operacionales que definen el proceso, así como aquellas que definen el nivel de protección brindada a los trabajadores, en las diferentes áreas que definen una Unidad Centralizada de Preparados Citostáticos, incluyendo la necesaria evaluación periódica de la cabina de seguridad biológica instalada, sobre la base de normativa referencial atingente. Este protocolo de evaluación y/o verificación debiera considerar aspectos asociados a:

- Determinación de velocidad y flujos de aire movilizados por componentes del sistema de control aerodinámico existente (en condiciones de operación), que debiera incluir:

- Test de uniformidad o velocidad del aire.

- Test de integridad o estanqueidad de filtros absolutos.

- Test de recuento de partículas (no viables, según familia ISO 14.644).

- Test de humo o similar, para demostrar unidireccionalidad del flujo de aire.

- Determinación de renovaciones/hora.

- Protección de áreas críticas por medio de diferencial de presión, incluyendo:

Test de integridad estructural del nivel de contención.

- Medición de niveles de iluminación y luminancia.

- Medición de niveles de ruido.

- Medición de temperatura ambiental.

- Medición de humedad relativa.

\section{Nivel de Riesgo en relación al Análisis de las Condiciones Existentes}

man a la existencia de claras falencias respecto de la infraestrucLa situación observada, junto con ratificar la hipótesis inicial, revela que en el 93\% de las instalaciones participantes del estudio existen condiciones de riesgo no controladas, asociadas a una gestión no efectiva del personal, en relación a la dotación actual de trabajadores, horas de la jornada destinadas a actividades de preparación y una creciente demanda poblacional por este tipo de prestación; factores que se suman a la existencia de claras falencias respecto de la infraestructura de control capaz de dotar y/o crear las condiciones de contención mínimas necesarias; efecto asociado a errores respecto de la selección y mantención de los medios que aseguren la calidad del proceso y control de la exposición, en particular aquellos asociados al estado de funcionamiento de las respectivas cabinas de seguridad biologicas instalñadas; errores interpretativos de la normativa por parte de proveedores, instaladores y terceros que prestan servicios de verificación y falta de control efectivo por parte de la autoridad y terceros responsables de cada Institución.

Proyecto realizado con el financiamiento de la Asociación Chilena de Seguridad, a través de la Fundación Científica y Tecnológica, FUCYT. 


\section{REFERENCIAS}

1. Falck K, Gröhn P, Sorsa M et al. Mutagenicity in urine of nurses handling cytoestatic drugs. Lancet. 1979; 1:1250-1. Letter.

2. P. J. M. Sessink, A. J. M. Verplanke, R. F. M. Herber, R. P. Bos, Occupational exposure to antineoplasic agents and parameters for renal dysfunction. Abstract Volume 69 Issue 3 (1997) pp 215-218.

3. Valanis V, Vollmer W, Labuhn K et al. Occupational exposure to antineoplasic agents and self-reported infertility among nurses and pharmacists. J Occup Environ Med. 1997; 39:574-80.

4. Evelo CTA, Bos RP, Peters JGP, Henderson PT (1986) Urinary cyclophosphamide assay as a method for biological monitoring of occupational exposure to cyclophosphamide. Int Arch Occup Environ Health 58:151-155.

5. Sessink PJM, Cerna M, Rossner P. 1994. Urinary cyclophosphamide excretion and chromosomal aberrations in peripheral blood lymphocytes after occupational exposure to antineoplasic agents. Mut Res. 309:193-199.

6. Ensslin AS, Stoll Y, Pethran A, Pfaller A, Römmelt H, Fruhmann G (1994). Biological monitoring of cyclophosphamide and ifosfamide in urine of hospital personnel occupationally exposed to cytostatic drugs. Occup Environ Med 51:229-233.

7. Kopjar N, Garaj-Vrhovac V. (2001). Application of the alkaline comet assay in human biomonitoring for genotoxicity: a study on Croatian medical personnel handling antineoplasic drugs. Mutagenesis Jan;16(1):71-8.
8. Standard NSF-ANSI 49-2011, International Standard for Biosafety Cabinetry. Biosafety Cabinetry: Design, Construction, Performance, and Field Certification. National Sanitation Foundation, 2011.

9. Norma General Técnica №25, Manipulación de Medicamentos Antineoplásicos en Farmacias de Hospitales. Departamento de Asesoria Juridica del Ministerio de Salud, Chile (1998).

10. Norma General Técnica N59, Manipulación de Medicamentos Estériles en Farmacias de Hospitales, Ministerio de Salud, Chile (2001).

11. Primary Containment for Biohazards: Selection, Installation and Use of Biological Safety Cabinets, U.S. Department of Health and Human Services, Public Health Service, Centers for Disease Control and Prevention and National Institute of Health, 2nd Edition, september 2000.

12. OSHA Technical Manual, Controling Occupational Exposure to Hazardous Drugs, Occupational Safety \& Health Administration (OSHA); january 1999.

13. Protection du Manipulateur, Cytotoxiques: Utilisation Pratique 3ème Éd., Centre National Hospitalier d'Information sur le Médicament, Dossier 1998, XIX, 2-3, 16. ISSN 0223.5242.

14. Quality Standard for the Oncology Pharmacy Service with Commentary. QUAPOS Published by the Institute for Applied Healthcare Sciences (IFAHS e.V.) and the German Society of Oncology Pharmacy (DGOP e.V.) as the result of the 11th North German Cytostatics Workshop (NZW), January 2003. 